\title{
Germanica
}

\section{Mein Name sei Gantenbein de Max Frisch : les visages de la Comédie et du Refus}

Mein Name sei Gantenbein de Max Frisch : les visages de la Comédie et du Refus

\section{Anne-Marie Baranowski}

\section{OpenEdition}

\section{Journals}

Édition électronique

URL : http://journals.openedition.org/germanica/1319

DOI : 10.4000/germanica.1319

ISSN : 2107-0784

\section{Éditeur}

Université de Lille

\section{Édition imprimée}

Date de publication : 30 juin 1998

Pagination : 65-77

ISBN : 9770984263203-22

ISSN : 0984-2632

Référence électronique

Anne-Marie Baranowski, « Mein Name sei Gantenbein de Max Frisch : les visages de la Comédie et du Refus », Germanica [En ligne], 22 | 1998, mis en ligne le 31 janvier 2012, consulté le 06 octobre 2020 URL : http://journals.openedition.org/germanica/1319; DOI : https://doi.org/10.4000/germanica.1319

Ce document a été généré automatiquement le 6 octobre 2020.

(c) Tous droits réservés 


\title{
Mein Name sei Gantenbein de Max Frisch : les visages de la Comédie et du Refus
}

\author{
Mein Name sei Gantenbein de Max Frisch : les visages de la Comédie et du \\ Refus
}

Anne-Marie Baranowski

1 La problématique de Mein Name sei Gantenbein se fonde sur le désir et la peur liés à l'amour et la mort pour entraîner une enfilade de dédoublements au niveau des personnages ainsi qu'une polarisation, assortie d'une imbrication étroite entre le vécu et l'imaginaire. Un seul article ne saurait rendre compte des aspects et facettes multiples de cette œuvre brillante et complexe, parue en1964 et constituant après Stiller (1954) et Homo Faber (1957) le troisième volet de la « trilogie zurichoise »; nous la considérerons donc essentiellement sous l'angle de l'homologie existant entre Enderlin et Gantenbein dont les aventures alternent de manière à former un semblant d'intrigue.

\section{Enderlin ou l'isolement dans la fuite}

Cette intrigue ne se dévoile que très progressivement au lecteur attentif: l'universitaire zurichois Felix Enderlin s'engage dans une liaison théoriquement éphémère, mais susceptible de prendre une place importante dans sa vie, ce qui le plonge dans des abîmes d'indécision et de perplexité. Il s'est rendu à un rendez-vous d'affaires pour y rencontrer un nouveau partenaire qu'il n'avait encore jamais $\mathrm{vu}$; retenu à Londres et ne pouvant le prévenir à temps, celui-ci a dépêché sa femme au bar où devait avoir lieu ce rendez-vous. Le hasard a jeté les dés de ce qui commence à se profiler sous les traits de la nécessité lorsque, incapable de trancher entre réticence et désir, Enderlin se laisse entraîner : ne pouvant se résoudre à partir, il est demeuré un long moment en compagnie de la jeune femme, puis a accepté de l'accompagner à 
l'opéra le soir-même, au lieu de quoi ils passent la nuit ensemble au domicile de celle-ci. Le dilemme se pose avec une acuité décuplée le lendemain matin, car cette femme infidèle, facile - anonyme presque d'un bout à l'autre du roman - a fait surgir des potentialités encore inexistantes la veille.

3 Enderlin est dévoré par l'anxiété, car à l'auto-analyse se mêle la puissance du fantasme : l'instance analytique prend le pouls et fait le point de la situation, recense des implications que l'imaginaire se met à développer comme autant de scénarios cinématographiques, repoussant à l'infini les limites de la rationalité. Tant qu'il n'agit pas, tout peut arriver. Ce dynamisme de l'imaginaire, par opposition à la passivité effective, se retrouve dans l'un des passe-temps favoris d'Enderlin quand il voyage: suivre des inconnus au cœur d'une ville étrangère, sans désir de les aborder, dans la simple intention de permettre aux associations d'idées engendrées par leur apparence, de s'éveiller et prendre forme. Ainsi se dessine une persona fantomatique, fugace, permettant le cas échéant de poser l'équation du présent et du passé, de l'inconnu et du sujet imaginaire : quittant de bonne heure le domicile de sa maitresse, Enderlin, qui porte un habit de soirée, se souvient d'avoir suivi à Paris un homme de belle apparence, en habit de soirée, prenant un café au petit matin dans un bistrot. Aujourd'hui, les rôles sont inversés, l'inconnu de Paris devient un miroir, mais un miroir à la fois opaque et sans tain, image d'une expérience stérile :

Je sais très bien que cela ne mène à rien; tôt ou tard, cet homme que je suis du regard va disparaitre dans l'embrasure d'une porte ou bien il va faire soudain signe à son taxi [...] Alors, je n'aurai plus qu'à me faire reconduire à mon hôtel pour me jeter sur mon lit sans avoir enlevé mes vêtements ni mes chaussures, épuisé d'avoir tant couru pour rien... C'est une manie que j'ai ${ }^{1}$.

L'anonymat rend les hommes interchangeables; l'opacité du silence et l'absence de regard engendrent une liberté infinie car indifférenciée. Il en va tout autrement en amour, où l'altérité cesse d'être muette, indifférente. L'imaginaire impitoyable anticipe en effet le devenir du couple avant même que celui-ci n'apparaisse ; le devenir fictif menace la réalité potentielle. Tout serait fini avant d'être commencé en raison de l'inévitable usure apportée par le temps et l'habitude :

Vous êtes un couple, intimement persuadé que vous ne vous perdrez plus jamais [...] Vous vous comprenez sans être obligés d'être d'accord. Vous êtes vivants, vous exprimez vos points de vue, mais vous connaissez vos corps de la même manière que vous connaissez vos meubles [...] Maintenant n'est pas «maintenant » mais «toujours» [...] Vous êtes seuls dans une maison, vous deux, mais vous l'êtes souvent, si souvent. Il n'y a rien à faire. La vie conjugale vous a rattrapés. Et vous vous donnez un baiser qui ressemble à un point à la fin d'une phrase. Vous êtes remplis du désir, non pas de l'un pour l'autre, car vous êtes là, après tout, vous êtes remplis du désir de sortir de vous-mêmes et de vous dépasser, mais ensemble ${ }^{2}$.

L'anonymat, dans la solitude et la liberté qu'il apporte, permet àEnderlin de garder un semblant d'autonomie au cœur de l'univers social et mondain où il évolue. Universitaire suisse, libéral, il se situe politiquement au centre-gauche. Son cercle d'amis et de relations demeure anonyme, - le seul nom mentionné est celui de Burri, un ami médecin - et n'apparaît qu'à travers des fragments de conversations rapportées au style indirect, assortis de quelques détails matériels. Grâce à cette surimposition de petites touches à la manière impressionniste, le lecteur conçoit une image assez nette de cet univers caractérisé par l'aisance matérielle et financière d'une classe moyenne aux revenus confortables, royalties ou émoluments de professions souvent libérales médecin, architecte, critique littéraire ou universitaire - profitant pleinement de la 
prospérité des années cinquante et soixante. Ce monde aimable et superficiel, cultivé parce qu'il a des opinions sur les noms de la littérature alors en vogue - Jünger, Joyce, Kafka - dispose de temps et d'argent pour des loisirs qui occupent une place importante dans sa vie. Les réceptions sont fréquentes dans un décor toujours interchangeable: vastes appartements ou villas cossus avec balcon, terrasse, piscine. La tradition et la modernité toutes deux confortables y fraternisent dans la discussion amicale : Mozart et jazz; whisky et cigare. Les destinations de vacances sont aussi révélatrices que stéréotypées : avide de soleil et de mer bleue, la bourgeoisie suisse privilégie l'Italie, l'Espagne, le Midi de la France, voire la Grèce. Signe de statut social, les voyages en avion y prennent un relief particulier. C'est un monde insipide, sans méchanceté ni bonté, où tout finit par se savoir sur les uns et les autres, où tout est interchangeable : lectures, domiciles, amants et maîtresses.

6 Sous des dehors d'urbanité affable, Enderlin y évolue à la manière d'une forteresse assiégée. Si la latitude qu'il accorde à son imaginaire lui permet de sauvegarder une indépendance discrète, la présence d'une femme à ses côtés le clouerait sous le regard d'autrui, l'engluant non seulement dans les commentaires mais aussi dans les stéréotypes et clichés qui défigurent la relation amoureuse ${ }^{3}$. Rendre cet amour public équivaut également à l'inscrire dans la durée, assujettissement à la temporalité porteuse de mort.

$7 \mathrm{Au}$ moment où débute cette liaison inattendue, Enderlin se trouvait déjà à un autre carrefour, professionnel celui-là et lui aussi lourd de conséquences : quitter l'Europe pour enseigner à Harvard où on lui a proposé un poste, est une consécration mais qui, confortant son statut universitaire, l'y assujettit encore davantage. La réussite est le deuil potentiel de la liberté. Aussi longtemps qu'il tergiverse, qu'il tarde à confirmer son acceptation, Enderlin garde quelque latitude.

Or cette liberté relative est menacée d'une interruption radicale lorsque le hasard intervient une troisième fois. Convalescent après une maladie de toute évidence assez sérieuse mais dont on sait simplement qu'elle a nécessité un séjour en clinique, Enderlin croit lire sur son dossier qu'il ne lui reste plus que douze mois à vivre. De fait, le lecteur apprend très vite qu'il s'agit là d'un quiproquo - le dossier était celui d'un autre patient - le médecin ne sait rien de cette erreur et Enderlin est le seul à se croire au terme de sa vie, subissant l'irruption, apparemment simultanée ou presque, de l'amour et de la menace de la mort dans son existence. Une gradation intervient dans l'altérité : partir pour les U.S.A. implique un changement de vie qui nécessite des ajustements mais ne constitue en somme que la continuation sous d'autres cieux, de l'activité professionnelle exercée jusqu'à présent en Suisse. Le début d'une liaison est plus ambigu ; les commencements sont uniques, la femme que l'on découvre l'est peutêtre aussi, mais avec le temps et la routine viennent les stéréotypes; de surcroît, Enderlin a de nombreuses conquêtes à son actif, ce qui relativise jusqu'à la trivialité cette liaison nouvelle. Il en va tout autrement de la mort, événement unique, crucial, oméga de la vie humaine, faisant peser sur le roman une menace qui imprime un tour très particulier à celui-ci.

9 Frisch introduit une zone intermédiaire entre Enderlin et Gantenbein, une sorte de no man's land marqué par les notions de hasard et de destin mêlées, sous forme de monologues intérieurs, tenus par un sujet anonyme, sur le thème de l'accident de voiture. Le personnage dérape et évite de justesse une catastrophe, ou bien il se remet d'un accident qui l'a rendu aveugle: ayant frôlé la mort, il voit sa vie marquée 
irrémédiablement par cette expérience, similaire à celle d'Enderlin qui se pense condamné, réagit en fonction de ce traumatisme intérieur et survit. L'identité du personnage n'est jamais révélée. Il pourrait s'agir d'Enderlin tentant de se suicider, imaginant un suicide potentiel: toute hypothèse est vraisemblable car elle ne sera jamais confirmée ni démentie. Par contre, l'idée à retenir de ces épisodes sybillins est celle d'une rencontre " active » avec la mort qui ne vient plus solliciter l'individu par la maladie ; c'est lui qui la rencontre au cours d'un déplacement comme s'il se dirigeait vers une destination choisie que seul le hasard lui fait manquer ${ }^{4}$.

La cécité présente également de multiples connotations. Elle aussi est une forme de mort, le sujet se trouve dissocié du monde qu'il ne voit plus, coupé de l'extérieur et projeté dans la nuit ou dans un univers totalement subjectif. Dans le langage courant, la justice, le destin, le malheur sont dits aveugles, comme est aveugle celui qui ferme les yeux sur ce qu'il refuse de voir. Frisch introduit donc de manière métonymique la notion de rupture de la vie, de mise en jeu de l'existence. La vie reproduit, une fois de plus, le schéma du coup de dés fondé sur le hasard et engendrant la nécessité. Il est possible de biaiser avec l'amour, de le dénier, mais la mort exige d'être prise en compte et quiconque aura un jour fait l'expérience de son imminence - réelle ou supposée - ne pourra plus jamais être le même.

\section{Survivre par le mensonge : Gantenbein}

Gantenbein surgit comme ex nihilo au début de l'œuvre : un homme essaie des lunettes de soleil chez un opticien sans parvenir à arrêter son choix, lorsqu'il en avise une paire aux verres fort sombres, des lunettes d'aveugle, qu'il achète en dépit des objections de la vendeuse. Il ne lui reste qu'à obtenir d'un médecin assermenté l'attestation lui donnant droit à l'obtention du brassard jaune destiné à marquer son état; tout s'accomplit sans le moindre problème: Gantenbein a fait, par hasard semble-t-il, le choix d'une certaine forme d'existence dans laquelle il se glisse avec la plus grande facilité.

12 Son récit, à la première personne, présente une succession de tableaux souvent drôles, réminiscents des comédies américaines et italiennes; il partage la vie d'une actrice, Lila, dont certaines caractéristiques laissent entendre qu'elle est l'image fantasmée de la maîtresse d'Enderlin : les mêmes yeux bleus, les mêmes cheveux noirs, l'une porte le jour de sa rencontre avec Enderlin un tailleur jaune qui réapparait dans la garde-robe de Lila. L'œuvre est cependant trop complexe pour autoriser l'assimilation pure et simple de Gantenbein à Enderlin, tous deux figurent effectivement une forme de dédoublement, mais ils sont bien davantage. Il est plus modeste et plus juste de dire que, considéré sous l'angle de sa relation avec Lila, Gantenbein est la projection, doublée d'une réécriture simultanée, des incertitudes qui tenaillent Enderlin. Le couple qu'il forme avec Lila se présente comme une série d'extrapolations, d'anticipations et de variations sur celui que formeraient éventuellement Enderlin et sa maîtresse. C'est aussi une réflexion sur la vie conjugale et l'amour en général. Il y a alors dédoublement dans la mesure où Enderlin figure la réalité « vécue » analytiquement et Gantenbein la transposition ludique, la dramatisation de cette réalité, enrichie d'une dimension de comédie satirique en même temps que d'une réflexion implicite sur la notion de «voir» et de « regard». Gantenbein est le doublet-complémentaire d'Enderlin, mais 
aussi la réponse - impraticable - aux questions qui demeurent sans réponse pour ce dernier.

13 Avec Lila et Gantenbein, l'univers social et mondain redevient omniprésent, c'est le même que celui d'Enderlin. Cependant il n'est plus restitué de manière oblique, indirecte, mais apparaît à travers la comédie de mœurs, le sketch satirique, inaccessibles au sérieux et analytique Enderlin. À Gantenbein par contre tout est permis : usurpant une infirmité terrible, il a déjà commis une forme de sacrilège et, évoluant dans un univers frelaté, il devient pour le lecteur un aimable iconoclaste. Sa cécité feinte lui permet une existence paradoxale, le rendant à la fois central et marginal: tout le monde s'empresse de l'aider et personne ne se gêne devant lui. " Homme au foyer ", il est entretenu par Lila, ce qu'il reconnaît sans la moindre gêne, il s'occupe impeccablement de leur vie matérielle, dont une actrice frivole, bohème et gâtée ne soupçonne même pas les multiples et complexes détails. Ce couple non conventionnel est donc heureux, à la grande surprise de tous les amis qui tablaient sur un divorce rapide et médiatique. Or, la cécité constitue un facteur positif permettant à chacun des époux de jouer un rôle, d'offrir à l'autre une comédie inédite: Gantenbein laisse toute latitude à Lila, ne lui révélant jamais qu'il a découvert qu'elle lui est infidèle. Lorsqu'il va la chercher à l'aéroport après un tournage à l'étranger, il la voit toujours descendre de l'avion en compagnie du même homme qui s'éclipse peu avant d'arriver à sa hauteur, en conséquence de quoi Gantenbein n'a jamais vu le visage de son rival. Dans l'optique de Frisch, la sincérité, même partielle, est en effet impossible au sein du couple, en raison de la dimension fantasmatique de l'amour et des réactions innées aux deux sexes :

Contrainte par la vanité de l'homme à jouer la comédie, la femme feint de fondre de plaisir, même quand ce plaisir fait défaut ; l'homme ne sait jamais avec certitude ce qui s'est vraiment passé pour la femme ; c'est l'homme qui se dévoile, ça le rend méfiant... Avant qu'on l'aime, la femme est un être humain, elle l'est parfois aussi après ; dès qu'on l'aime, elle est un miracle, donc impossible à retenir ${ }^{5}$.

14 Les amants sont alors implicitement contraints au mensonge. Cette analyse désespérante et sèche est le fait d'Enderlin. Gantenbein va se charger de la développer, de l'illustrer, et le récit syncopé qu'il donne de son mariage avec Lila est la démonstration ludique, ironique, la mise en scène cinématographique de cette proposition. Gantenbein trompe également Lila, par son mensonge, mais aussi avec Camilla Huber, manucure de son état, call-girl en réalité. Cette liaison n'a rien de sexuel : il assouvit sur Camilla son besoin de raconter des histoires, ce à quoi il excelle. Ces contes sont autant de variations, paraboles, métaphores, méditations sur le thème de l'amour et du couple, faisant intervenir la jalousie, le chagrin: tout ce qu'il a soigneusement évacué de sa fictive réalité. Sa vie avec Lila apparaît à travers les scènes de comédie qu'il livre au lecteur comme quelque chose de pétillant, de piquant qu'il faut goûter sans trop le prendre au sérieux; la dimension ludique, l'humour dédramatisent la vie en épinglant les travers, les ridicules, les faussetés de la comédie humaine. En témoigne cet épisode où l'« aveugle » Gantenbein livre victorieusement un combat non déclaré à la directrice d'une boutique élégante désireuse de faire acheter à Lila un tailleur rouge qui ne lui va pas du tout :

Je demande : « Est-ce le [tailleur] jaune? » « Non, dit-elle, c'est le rouge [...]».

Aveugle, Gantenbein se rappelle toutes sortes de rouges [...] Il dit ne se rappeler qu'un seul rouge qui ne lui irait pas: une espèce de rouge sans profondeur, artificiel, chimique : rouge limonade.

«Oh, non, dit-elle, ce n'est pas un rouge limonade». 
J'attends en fumant.

« Non, dit la dame, certes non ».

J'attends en fumant.

«Ou bien, trouvez-vous, dit Lila en jetant un coup d'œil dans le miroir, que c'est un rouge limonade? «Allons donc! ». Dans le miroir, je vois Lila hésiter.

Je pose d'autres questions auxquelles Lila répond avec une assurance qu'elle est incapable de soutenir face au miroir. Par exemple :

«Et n'est-il pas trop dépouillé ?»

Il est bourré de fioritures.

«Oh non, dit Lila, pas du tout ».

Je fume.

«S'il vous plaît, dit Lila à voix basse, essayons encore une fois le jaune $»^{6}$.

Pourtant le jeu lui-même ne saurait tout conjurer et, à côté de l'adultère devenu élément de comédie, figure un autre épisode dont les protagonistes portent, ironiquement, les noms de Philémon et Baucis : Philémon - Gantenbein - découvre que Lila-Baucis reçoit très régulièrement des lettres en provenance du Danemark, dont elle ne lui parle pas. Il en subtilise une et découvre qu'il s'agit de lettres d'amour : apparaît la passion romantique inopportune, nourrie d'éloignement, de séparation et d'interdits. Suit une longue analyse de la jalousie, d'une crise conjugale mettant le couple au bord du divorce. L'union est sauvée de justesse, mais c'est la grossesse qui se profile à présent, continuation logique, implacable du couple. Lila annonce qu'elle va être mère. Gantenbein est dubitatif quant à sa paternité, mais Béatrice vient au monde et son père l'élève pendant que Lila poursuit sa carrière. Défilent toutes les étapes de la vie d'un enfant, sorties tout droit du livre d'images de la famille modèle. Puis Béatrice grandit et commence à se bâtir une vie personnelle où les parents deviennent simplement utilitaires :

Un jour dans l'avenir (mais cela n'arrivera peut-être même pas) ils seront dans un café, le père et la fille qui est à présent une demoiselle, et une demoiselle dans l'embarras [...] un échec scolaire [...] pendant que Gantenbein fume, non sans fierté à l'idée de devoir son existence à cette superbe créature qui a subi un échec scolaire et requiert son aide tout en mangeant des pâtisseries [...] Béatrice n'a pas besoin de ses consolations, mais de sa signature et de l'argent nécessaire à la fréquentation d'une meilleure école ; ce qu'elle demande est clair et net; elle ne demande pas de camaraderie [...] Comment un enfant, si gentil soit-il, pourrait-il s'imaginer qu'un père a également ses misères ? [...] Il appartient au passé et le présent, ce n'est pas le père avec sa fille, mais la fille ${ }^{7}$.

Gantenbein fait l'expérience d'une vérité immémoriale : les enfants, même de fiction, sont la mort de leurs parents. La paternité, comme l'amour, laisse le goût de cendre de toutes les relations, tous les sentiments humains menés à leur terme. Au commencement était l'absolu, au commencement seulement. Situé en fin d'ouvrage, l'épisode de la paternité est l'une des dernières constructions fantastiques de Gantenbein dont la trajectoire imaginaire arrive à son terme ${ }^{8}:$ il y a eu le début de sa vie avec Lila, arrachée de main de maître à un amant de très longue date; cette vie commune qui apparaît à travers autant de tableaux et où le sérieux du réel finit par l'emporter sur la fantaisie de la fiction et de l'imagination ; la rupture enfin. Celle-ci est due précisément à ce sérieux de la vie qui est parvenu à s'infiltrer dans l'imaginaire et à le corrompre. Dès lors, Gantenbein n'attend qu'une occasion de mettre fin à ce roman, occasion qui s'offre à lui de manière paradoxale : il découvre un jour que l'inconnu de l'aéroport a disparu ; Lila a cessé de le tromper et leur couple perd ce déséquilibre qui lui avait permis de durer si longtemps. La comédie doit s'arrêter lorsqu'elle ne peut 
plus être réciproque 9 . Refusant de se rendre à la normalité, Gantenbein avoue sa supercherie à Lila qui le chasse après lui avoir fait une scène terrible, théâtrale.

Cette rupture en fin de roman renvoie au chapitre liminaire précédant l'apparition d'Enderlin et de Gantenbein: un homme pénètre dans un appartement qui fut visiblement le sien. Un couple y a vécu récemment, mais tout indique la séparation : quelques reliefs d'aliments gâtés dans le réfrigérateur; des housses sur les meubles, des vêtements féminins dans les placards. Lila et Gantenbein auraient pu vivre là, ce que le lecteur ignore encore. Or, cet appartement abandonné renvoie également à Enderlin, car l'homme est un fugitif très particulier, il s'est échappé entièrement nu d'une clinique, après avoir pris un bain - interdit par le médecin - et effrayé l'infirmière de service. La chronologie est inversée : le début de cette scène arrive bien plus tard, lorsque Frischaborde le séjour en clinique d'Enderlin. Les deux moments du texte sont liés par l'idée de la mort et la présence de l'infirmière, Elke. Le lien unissant ces deux épisodes qui apparaissent comme deux pièces délibérément disjointes du même puzzle est extrêmement complexe. L'épisode initial - fuite de l'homme nu - est la suite fantasmée du second, qui suit immédiatement le moment oùEnderlin croit apprendre qu'il est condamné. Le désespoir provoque en lui une brusque montée de désir et de fantasmes sexuels liés à la féminité, dont l'unique représentante est alors Elke; son physique ingrat ne représente que : «[...] Le fantasme d'un malade qui n'a plus le choix, d'un homme sans force qui ne fera plus long feu $»^{10}$.

Par contre, les images transgressives du désir s'adressent à la Féminité :

Et il ne pense qu'à leur ventre; pénétrer dans leur ventre [...] leur bouche et sa langue dans leur bouche, quand leurs visages se fondent et se confondent [...]. Des ventres, des lèvres, des cuisses, des cheveux, des seins, des yeux qui se rétrécissent pour ne plus former que des fentes, et des ventres, des ventres, des ventres de femme $[. . .]^{11}$.

19 Or, la suite est très différente du fantasme érotico-intellectuel, c'est en effet une véritable mise en scène qui prélude à la fuite et qui commence lorsque l'homme se fait couler un bain ; Elke, qui l'a surpris, devient simultanément image de la Femme et de la mort :

[...] Mais voilà que l'homme nu lui barre le chemin et comme il n'a rien à ce moment-là pour se couvrir devant la jeune fille, il recourt à une plaisanterie : Je suis Adam ! [...] tandis qu'il la regarde comme s'il voyait Elke pour la première fois. Une jeune fille aux yeux gris ou verts. Il la prend par les épaules. Une jeune fille à la chevelure roussâtre et aux grandes dents [...] Eve prenant le visage d'une infirmière de nuit, d'une paysanne venue de la Baltique pour faire ses études, aux yeux verts et à la mâchoire de cheval ${ }^{12}$.

La situation de départ est ainsi remise en cause a posteriori, la chronologie événementielle qui s'était dégagée progressivement et à grand'peine est ébranlée : la rencontre et le début de la liaison avec la jeune femme anonyme ne seraient peut-être pas un commencement, mais plutôt un souvenir, évoqué à travers les brumes de la fièvre et de la maladie. La liaison pourrait être déjà terminée et le couple LilaGantenbein deviendrait le produit du fantasme d'un malade revenant par la mémoire sur ce qui a cessé d'exister. Toutes les hypothèses sont cohérentes dans la mesure où elles sont assurées de ne pas rencontrer de conclusion. Le « roman » fait coexister des intrigues partielles qui évoluent en parallèle les unes des autres et simultanément de manière à former une mosaïque ou un kaléidoscope, kaléidoscope brillant et faussement aimable où les jeux de l'esprit ne dissimulent qu'imparfaitement l'inanité, 
la vacuité intime et la désespérance. L'homme élude constamment toute rencontre vraie avec l'altérité féminine, construisant et animant des simulacres gracieux chargés d'accompagner et de distraire son imaginaire. Par contre, lorsqu'il est confronté à sa disparition physique, la féminité éludée prend sa revanche. Elle est devenue le doublet de la mort dont elle partage le caractère absolu. Elle en est en même temps l'antithèse. Le sein maternel et la tête hideuse du cheval chtonien s'imbriquent inextricablement au moment où le désir et l'illusion se dépouillent devant la fin de la chair, le moment où l'intellectuel esthète saisit intuitivement qu'il va mourir sans avoir su vivre.

\section{NOTES}

1. Mein Name Sei Gantenbein, 1964. Suhrkamp, Frankfurt/Main, p. 8-9.

2. Ibid, p. 123.

3. «Dès que l'on forme un couple, le genre humain semble n'être qu'une seule et même famille : tout le monde connaît tout le monde d'une façon ou d'une autre [...]» (p. 117).

4. Associée à la maladie, la figure chtonienne du cheval, emblème de la mort, prélude au roman à travers une vision hallucinée, délirante, celle d'un malade anonyme - Enderlin ou quelqu'un d'autre. Le monde aseptisé de la clinique, efficace et moderne, qui tente d'évacuer la mort, est impuissant à contenir l'émergence de l'innommable, archétypal et tôt ou tard inévitable. Dans cette vision, la paroi rocheuse que le malade découvre de sa fenêtre s'entrouvre, laissant apparaître un cheval qui tente en vain de sauter : « [...] Une tête de cheval aux yeux écarquillés, la mâchoire écumante, hennissant mais sans bruit [...] une tête exprimant une panique mortelle [...] la tête de cheval rentre sans bruit dans le rocher qui se referme silencieusement, sans montrer la moindre fissure ». Le cheval devient ensuite cheval-vapeur et l'automobile destrier moderne.

5. Mein Name Sei Gantenbein, op. cit., p. 189.

6. Mein Name Sei Gantenbein, op. cit., p. 85.

7. Ibid., p. 277-278.

8. La verve imaginative ne s'épuise pas, le trait demeure précis et acéré, mais la lassitude du jeu apparaît en même temps que l'admission implicite de son inanité : «mais cela n'arrivera peutêtre même pas ".

9. La vie conjugale telle que la conçoit Gantenbein requiert une part d'opacité qui soustrait l'Autre au regard. Savoir qu'il y a une part de l'Autre que l'on ne peut, que l'on ne doit pas connaître et vice-versa, est la condition nécessaire à la durée d'un couple, lui permettant de s'inscrire dans la durée tout en échappant aux cliché et à la routine mortels, engendrés par le trop connu. La vie quotidienne dévoile, le regard perce l'intimité, mais le mensonge restitue à l'autre son mystère, sa capacité de surprendre, de jouer avec le partenaire. La réciprocité de jeu va de pair avec le secret bien gardé : «Si Lila savait que je vois, elle douterait de mon amour. L'homme et la femme ne deviennent couple qu'avec ce secret qu'ils se dissimulent l'un à l'autre ». C'est la condition qu'énonce Gantenbein au commencement de sa vie conjugale. Le contrepoint, mettant un terme à celle-ci apparaît avec cette constatation résignée, lorsque Gantenbein voit Lila descendre seule de l'avion: "À quoi bon poursuivre ce mariage d'aveugle? Lila ne me trompe pas. Alors ça ne sert à rien ».

10. Mein Name Sei Gantenbein, op. cit., p. 134.

11. Ibid., p. 135. 


\section{RÉSUMÉS}

Le héros de cette œuvre complexe, l'universitaire suisse Enderlin se trouve confronté à une série d'événements inattendus : on lui propose un poste à Harvard, il tombe amoureux et découvre ou croit découvrir - qu'il est atteint d'un mal incurable.

Incapable d'affronter la réalité. Enderlin se réfugie dans un univers fictif au sein duquel son double imaginaire, le faux aveugle Gantenbein, joue le rôle principal. Charmant, plein d'humour et dépourvu de scrupules, Gantenbein s'amuse aux dépens de l'élite intellectuelle dont il raille le conformisme et le caractère superficiel, ce que Enderlin n'a jamais eu le courage de faire.

Pourtant, la réalité l'emporte sur le monde de l'imagination. Le rêve d'Enderlin se brise lorsqu'il se découvre guéri mais seul. Gantenbein disparaît lorsque son existence de fiction se charge d'un conformisme et d'un ennui identiques à ceux qui caractérisent la vie vécue. La vie rêvée se mue en défaite, ce qui interdit à l'être humain toute perspective d'évasion.

In diesem vielschichtigen Werk sieht sich der vierzigjährige Akademiker Enderlin mit unerwarteten Ereignissen konfrontiert; er bekommt nämlich ein Lehrangebot nach Harvard, verliebt sich und nicht zuletzt entdeckt er, daß er unheilbar krank ist ; das glaubt er jedenfalls.

Unfähig, sich mit der Wirklichkeit auseinanderzusetzen, flüchtet sich Enderlinin eine Phantasiewelt, in welcher seinem fiktiven Doppelgänger Gantenbein die Hauptrolle zufällt. Voller Humor, charmant und skrupellos macht sich der angeblich blinde Gantenbein über die intellektuelle Elite lustig, bespottet ihren Konformismus und ihre Oberflächlichkeit, wozu Enderlin nie den Mut gehabt hat.

Doch auch die Phantasiewelt erliegt der Realität. Der Traum zerplatzt, wenn Enderlin entdeckt, daß er eigentlich geheilt ist, daß er aber vor den Trümmern seines Glückes steht. Auch Gantenbein entgeht dem Tod nicht, sein fiktives Leben wird allmählich von Konformismus und Langeweile überwältigt; nach und nach wird es ebenso seicht und uninteressant wie das Leben Enderlins. Jeder Ausweg bleibt dem Menschen verschlossen.

\section{AUTEUR}

ANNE-MARIE BARANOWSKI

Université du Littoral, Dunkerque 